\title{
sciendo
}

Current Issues in Pharmacy and Medical Sciences

Formerly ANNALES UNIVERSITATIS MARIAE CURIE-SKLODOWSKA, SECTIO DDD, PHARMACIA

\section{The development of HPLC-DAD method for determination of active pharmaceutical ingredient in the potassium 2-((4-amino-5-(morpholinomethyl)-4H-1,2,4-triazol-3-yl)thio) acetate substance}

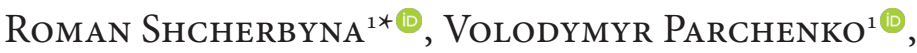 \\ BORIS VARYNSKYI ${ }^{2}$, ANDRIY KAPLAUSHENKO ${ }^{2}$ (๑)
}

${ }^{1}$ Department of Toxicological and Inorganic Chemistry, Zaporizhzhya State Medical University, Mayakovsky 26, 69035, Zaporizhzhya,
Ukraine
${ }^{2}$ Department of Physical and Colloidal Chemistry, Zaporizhzhya State Medical University, Mayakovsky 26, 69035, Zaporizhzhya, Ukraine

\section{ARTICLE INFO}

Received 08 February 2018

Accepted 30 April 2018

\section{Keywords:}

1,2,4-triazole,

HPLC-DAD,

an active pharmaceutical

ingredient.

\begin{abstract}
Derivatives of 1,2,4-triazole are actively researched by scientists and synthetic pharmacologists. The last studies have shown that potassium 2-((4-amino-5(morpholinomethyl)-4H-1,2,4-triazol-3-yl)thio)acetate with low toxicity series exhibits antioxidant and hepatoprotective properties. Therefore, the purpose of this work was to develop a method for determining the API in the potassium 2-((4-amino-5(morpholinomethyl)-4H-1,2,4-triazol-3-yl)thio)acetate substance using the method of high-performance liquid chromatography with diode array detection (HPLC-DAD). As a result of this work, it is shown that the developed method is specific and meets the requirements of linearity, accuracy and precision. The results of determining the contents of the API in real samples indicate that the method can be proposed to control the quality of the potassium 2-((4-amino-5-(morpholinomethyl)-4H-1,2,4-triazol-3-yl)thio)acetate substance.
\end{abstract}

\section{INTRODUCTION}

Creating new, original medicines is an incredibly complicated and expensive process. This axiom is well known to scholars who are actively engaged in this problem. In addition to pharmacological and toxicological documentation, the development and testing of methods for quality control of active pharmaceutical ingredients (APIs) is mandatory for implementation [1-3].

Derivatives of 1,2,4-triazole are actively researched by scientific teams around the world on the ability to manifest a wide range of biological effects [4-6]. Thus, in the scientific literature there is available data on their use as antimicrobial, anti-inflammatory, analgesic, antiepileptic, antiviral, antihypertensive, antimalarial, sedative, antihistamines, anti-TB drugs, etc. $[4,5,7,8]$. Interesting enough in pharmacological way, there are 2-((5-R-4-R1-4H-1,2,4-triazole-3-yl)thio) acetic acid salts, for which a wide range of pharmacological activity has been researched $[7,9,10]$. Of these salts,

\footnotetext{
* Corresponding author

e-mail: rscherbyna@gmail.com
}

2-((4-amino-5-(morpholinomethyl)-4H-1,2,4-triazol-3-yl) thio)acetate, which along with low toxicity, exhibits antioxidant and hepatoprotective properties [11].

Therefore, the development of a method for determination the API in the substance is one of the important steps towards the creation of a new medicinal product [12-14].

The purpose of this work is to develop a method for determining the API in the potassium 2-((4-amino-5(morpholinomethyl)-4H-1,2,4-triazol-3-yl)thio)acetate substance, using the method of high-performance liquid chromatography with diode-matrix detection (HPLC-DAD).

\section{MATERIALS AND METHODS}

As an object of research, potassium 2-((4-amino-5(morpholinomethyl)-4H-1,2,4-triazol-3-yl)thio)acetate (structure I, Fig.1) was used [15]. The study of the physical and chemical properties of the resulting compounds was carried out according to the methods presented in the State Pharmacopoeia of Ukraine (SPU) [16,17]. 


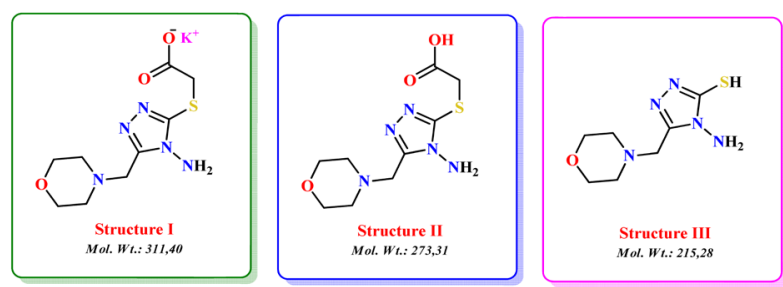

Figure 1. Structural formula of potassium 2-((4-amino-5-(morpholinomethyl)-4H-1,2,4-triazol-3-yl)thio)acetate (structure I), 2-((4-amino-5-(morpholinomethyl)-4H-1,2,4-triazol-3-yl)thio) acetic acid (structure II), 4-amino-5-(morpholinomethyl)- $4 \mathrm{H}$ 1,2,4-triazole-3-thiol (structure III)

The test was carried out by high-performance liquid chromatography with diode-matrix detection using an Agilent 1260 Infinity HPLC, with an Agilent 6120 mass spectrometer (electrospray ionization (ESI)).

\section{Materials}

Reagents: acetonitrile qualification "HPLC Super Gradient" (Avantor Performance Materials Poland S.A., Poland), formic acid (100\%) (AppliChem GmbH, Germany), ultra-high pure water $\left(18 \mathrm{M} \Omega\right.$ at $\left.25^{\circ} \mathrm{C}\right)$ was obtained by using the system for water Direct Q 3UV Millipore (Molsheim, France).

The substance of potassium 2-((4-amino-5-(morpholinomethyl)-4H-1,2,4-triazol-3-yl)thio)acetate (structure I) (API) was used. The compound was synthesized at Department of Toxicological and Inorganic Chemistry, Zaporizhzhya State Medical University.

\section{Chromatography conditions:}

- column - Ø 4.6 ×50 mm, RX-SIL, $1.8 \mu \mathrm{m}$, (Agilent Technology, USA);

- column temperature $-40^{\circ} \mathrm{C}$;

- mobile phase $\mathrm{A}-\mathrm{H}_{2} \mathrm{O}-0.1 \% \mathrm{HCOOH}$;

- mobile phase $\mathrm{B}-\mathrm{CH}_{3} \mathrm{CN}-0.1 \% \mathrm{HCOOH}$;

- eluent flow rate $-400 \mu \mathrm{l} / \mathrm{min}$;

- isocratic mode - mobile phase A: mobile phase B (20:80);

- sample volume - $1 \mu \mathrm{l}$;

- diode array detector $(\lambda=254 \mathrm{~nm})$.

The chromatography system suitability test. The number of theoretical plates $\mathrm{N}$ at the API peak should be $\geq 4,000$ (length of column $50 \mathrm{~mm}$ ).

Preparation of the mobile phase A. $1.00 \mathrm{~mL}$ of the formic acid was diluted to $1000.0 \mathrm{~mL}$ with a high purity water and was mixed.

Preparation of the mobile phase B. $1.00 \mathrm{~mL}$ of the formic acid was diluted to $1000.0 \mathrm{~mL}$ with acetonitrile and was mixed.

Preparation of a reference solution. $100 \mathrm{mg}$ (precise weight) of the standard potassium 2-((4-amino-5(morpholinomethyl)-4H-1,2,4-triazol-3-yl)thio)acetate was dissolved in $50 \mathrm{~mL}$ of high purity water, then it was diluted to $100.0 \mathrm{~mL}$ with water and was mixed thoroughly.

Preparation of the solution for chromatographic system suitability test. $100 \mathrm{mg}$ of the standard API sample was dissolved in $50 \mathrm{~mL}$ of a high purity water then was diluted to $100.0 \mathrm{~mL}$ with the water and was mixed thoroughly.
Preparation of the research solution. $100 \mathrm{mg}$ (precise weight) of the research sample of the potassium 2-((4-amino5-(morpholinomethyl)-4H-1,2,4-triazol-3-yl)thio)acetate substance was dissolved in $50 \mathrm{~mL}$ of high purity water, then it was diluted to $100.0 \mathrm{~mL}$ with the water and was mixed thoroughly.

The solution was then chromatographed for assessing the suitability of the chromatographic system $\mathrm{n}$ times, calculating relative standard deviation $(R S D)$ for peak API area; the chromatography was stopped when the obtained RSD value was less than the $R S D_{\text {max }}$ value or equal, that is given in the State Pharmacopeia of Ukraine (SPU) and SPU (Supplement 1) $[16,17]$ and calculated according to the European Pharmacopeia method 2.2.46 (SYSTEM SUITABILITY) [18] for the upper limit given in the definition of the individual monograph, minus 100 per cent $\mathrm{API}$ content $\mathrm{B}=2 \%$ (Table 1).

Table 1. The requirements for relative standard deviation $\left(R S D_{\max }\right)$

\begin{tabular}{|c|c|c|c|c|c|c|c|}
\hline $\mathrm{n}$ & 2 & 3 & 4 & 5 & 6 & 7 & 8 \\
\hline$R S D_{\max }$ & 0.32 & 0.84 & 1.2 & 1.48 & 1.72 & 1.93 & 2.11 \\
\hline
\end{tabular}

Alternately, the reference solution was chromatographed and the research solution was determined by the number of times (n). The retention time of the API peak should be about 4.4 minutes.

The API content in the substance potassium 2-((4-amino5-(morpholinomethyl)-4H-1,2,4-triazol-3-yl)thio)acetate X, $\%$, was determined by the formula:

$$
X=\frac{S_{x} \times m_{s t} \times P \times 100}{S_{s t} \times m_{x} \times(100-w)}
$$

where:

$S_{x}$-is the mean peak area of the API potassium 2-((4-amino-5-(morpho-linomethyl)-4H-1,2,4-triazol-3-yl)thio)acetate for the chromatogram of the research solution;

$S_{s t}$-is the mean peak area of the API potassium 2-((4-amino-5-(morpho-linomethyl)-4H-1,2,4-triazol-3-yl)thio)acetate for the chromatogram of reference solution;

$m_{s t}$ - is the weight of the standard API sample of potassium 2-((4-amino5-(morpholinomethyl)-4H-1,2,4-triazol-3-yl)thio)acetate, g;

$m_{x}$ - is the weight of the research sample of potassium 2-((4-amino-5(morpholinomethyl)-4H-1,2,4-triazol-3-yl)thio)acetate, g;

$P$ - is a content of the basic substance in the standard working sample, $\%$; $w$ - is a water content in potassium 2-((4-amino-5-(morpholinomethyl)$4 H$-1,2,4-triazol-3-yl)thio)acetate, $\%$.

\section{RESULTS AND DISCUSSION}

Reasoning of chromatographic quantitative determination conditions:

The compound which was studied is very hydrophilic with small retention (in the void volume) on reverse phase sorbent (C18). That is why the silica sorbent RX-SIL in HILIC (hydrophilic chromatography) mode was used. As the eluent, a mixture of water and acetonitrile (20:80) was used in the presence of $0.1 \%$ methanolic acid.

The substance which was synthesized firstly was studied by chromatography with DAD and MS detection.

Using a diode array detector at a wavelength of $254 \mathrm{~nm}$, we detected an impurity that was released at 2.7 minutes (Fig. 2). 


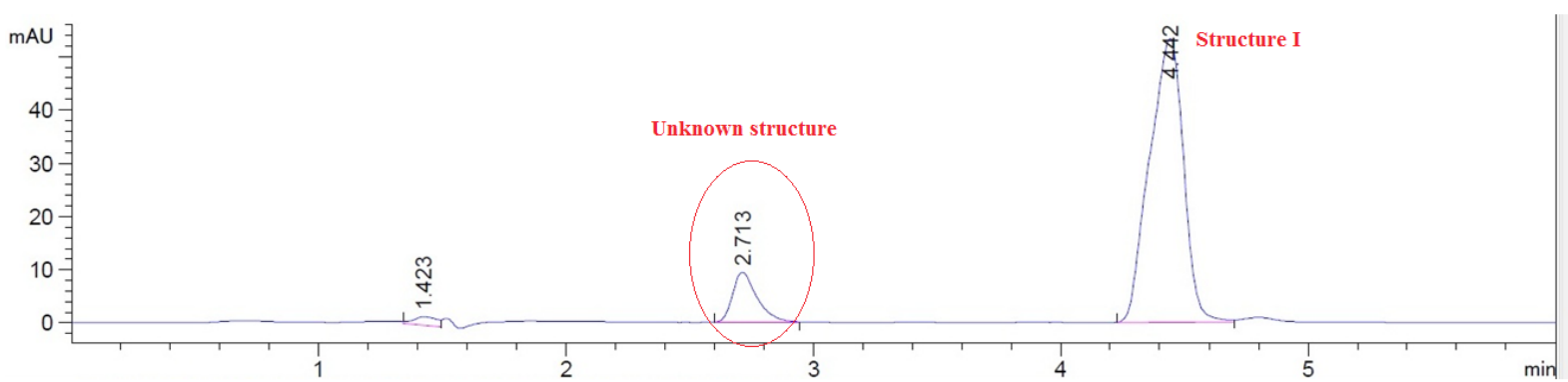

Figure 2. Chromatogram of $0.1 \%$ solution of the crude API (potassium 2-((4-amino-5-(morpholinomethyl)-4H-1,2,4-triazol-3-yl)thio) acetate) on DAD, $254 \mathrm{~nm}$

This unknown structure was not detected on the mass spectrometric detector in the positive ionization mode, at a voltage value of the $10 \mathrm{~V}$ fragmentor, scanning from 100 to $500 \mathrm{~m} / \mathrm{z}$, the gas velocity of the nebulizer of $10 \mathrm{~L} / \mathrm{min}$, gas pressure of the nebulizer of $60 \mathrm{psig}$, temperature of gasdehydrator (nitrogen) of $300^{\circ} \mathrm{C}$.

Therefore, in order to achieve the main objective of the work, the identification and removal of an impurity in potassium 2-((4-amino-5-(morpholinomethyl)-4H-1,2,4-triazol3-yl)thio)acetate (API) are needed.

It was proposed that the impurity might not fall within the standard scan range, so it was expanded to $\mathrm{m} / \mathrm{z} 50-500$. This decision did not lead to a positive result, which suggested that it is possible to detect the impurity in the negative mode (Mode name). As a result, the voltage on the fragmentor was increased to $50 \mathrm{~V}$, to increase the sensitivity of detection (with increasing voltage, the ion current increases and, accordingly, more ions enter the skimmer) [19].

It should be noted that these actions did not help in detecting the impurity either at $50 \mathrm{~V}$, or at $100,150,200$, $250 \mathrm{~V}$. At the same time, the intensity of the peak of the main component first grew, and then decreased, due to fragmentation of the molecule. These results allowed us to return to the positive ionization regime and increase the voltage on the fragmentator to $100 \mathrm{~V}$, and then to $150 \mathrm{~V}$. The impurity was, hence, detected and the intensity of the peak of this impurity increased. A characteristic peak of the ion with $\mathrm{m} / \mathrm{z} 216.1$ (Fig. 3) was then observed on the mass spectrum, which could correspond to the quasimolecular ion $\left(\mathrm{M}+\mathrm{H}^{+}\right)$of the initial product of the synthesis of
4-amino-5-(morpholinomethyl)-4H-1,2,4-triazole-3-thiol (structure III, Fig. 1).

Subsequently, an intermediate component of the synthesis of potassium 2-((4-amino-5-(morpholinomethyl)4H-1,2,4-triazol-3-yl)thio)acetate (API) - 2-((4-amino-5(morpholinomethyl)-4H-1,2,4-triazol-3-yl)thio)acetic acid (structure II, Fig. 1) was seen. As a result, the same impurity was observed, with the same $\mathrm{m} / \mathrm{z}$ and retention time.

Further, for the final identification of the impurity, an analysis was made of the starting component 4-amino-5(morpholinomethyl)-4H-1,2,4-triazole-3-thiol (structure III, Fig. 1). The resulting mass spectrum and retention time (Fig. 4) coincided with those for the impurity, which confirmed that this impurity is 4-amino-5-(morpholinomethyl)4H-1,2,4-triazole-3-thiol ( structure III).

The results obtained suggest that in the research sample, potassium 2-((4-amino-5-(morpholinomethyl)-4H-1,2,4triazol-3-yl)thio)acetate (API) yield of $2.713 \mathrm{~min}$ (Fig. 2) corresponds to the starting product of the synthesis, namely 4-amino-5- (morpholinomethyl)-4H-1,2,4-triazole-3-thiol (structure III, Fig. 1). Therefore, potassium 2-((4-amino5-(morpholinomethyl)-4H-1,2,4-triazol-3-yl)thio)acetate (API) was obtained by double recrystallization in which no impurities of the starting products were detected, which made it possible to use this substance to prepare a reference solution (Fig. 5).

\section{Determination of the linear range of the method}

Linearity was determined by introducing into the chromatographic system $0.8 ; 0.85 ; 0.9 ; 0.95 ; 1 ; 1.05 ; 1.1$;

a)
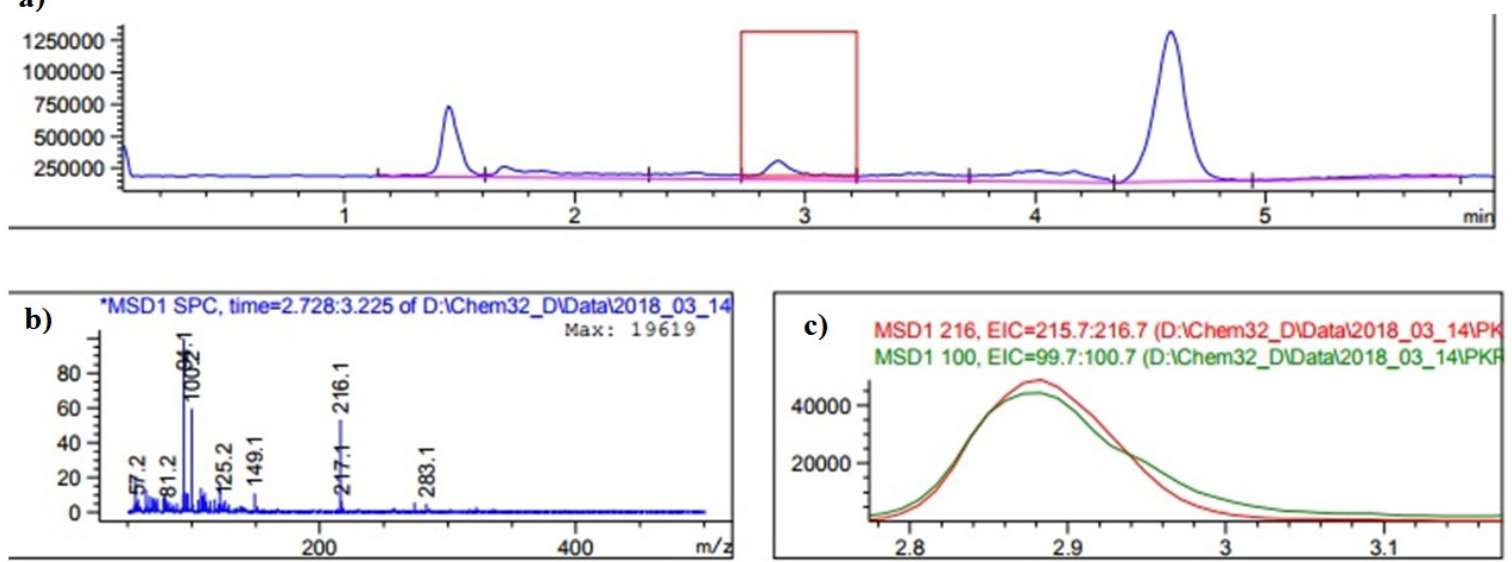

Figure 3. TIC (a) chromatogram of $0.1 \%$ solution of the API (potassium 2-((4-amino-5-(morpholinomethyl)-4H-1,2,4-triazol-3-yl) thio)acetate) at fragmentation voltage $150 \mathrm{~V}$, mass spectrum (b) and EIC chromatogram (c) of impurity 

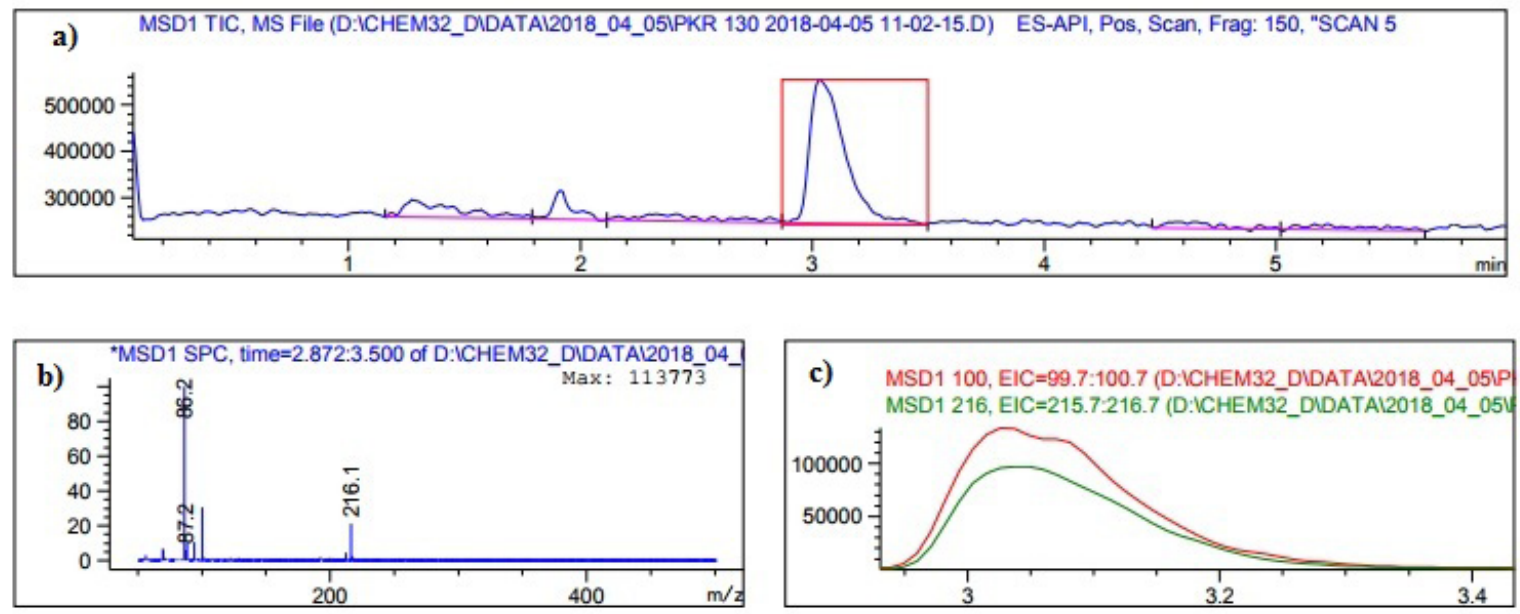

Figure 4. TIC (a) chromatogram of $0.01 \%$ solution of the 4-amino-5-(morpholinomethyl)-4H-1,2,4-triazole-3-thiol at fragmentation voltage $150 \mathrm{~V}$, mass spectrum (b) and EIC chromatogram (c)

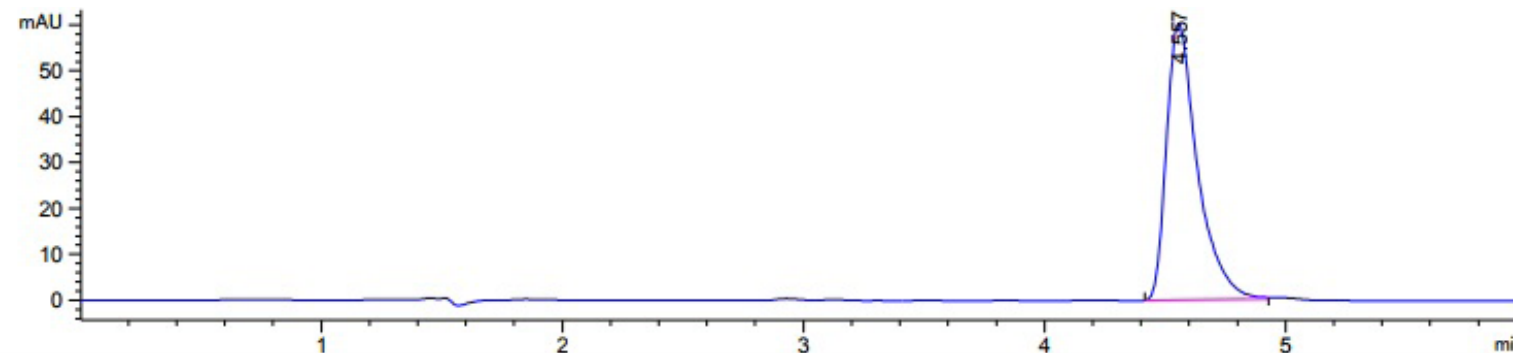

Figure 5. Chromatogram of a $0.01 \%$ reference solution of potassium 2-((4-amino-5-(morpholinomethyl)-4H-1,2,4-triazol-3-yl)thio) acetate at $254 \mathrm{~nm}$

$1.15 ; 1.2 \mu 1$ of the standard potassium $2-((4-a m i n o-5-$ (morpholinomethyl)-4H-1,2,4-triazol-3-yl)thio)acetate solution and the graphic dependence of the peak area on the amount of the introduced sample at 204, 210, $254 \mathrm{~nm}$.

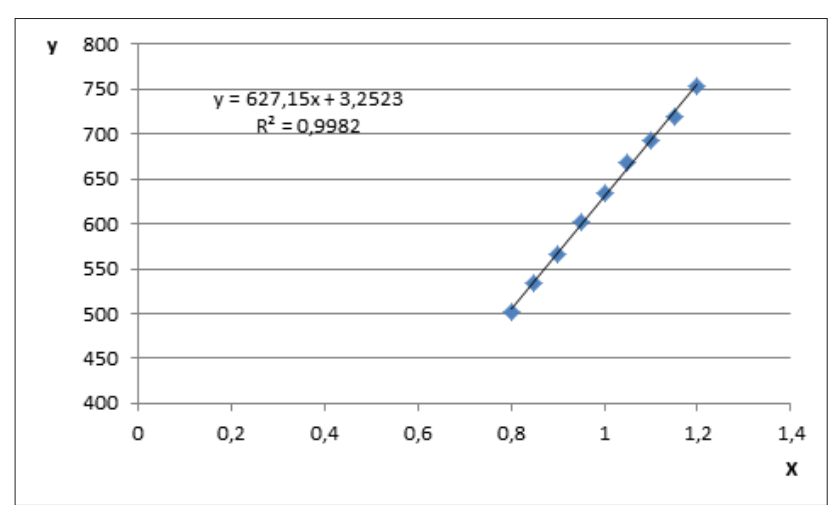

Figure 6. Graphic dependence of peak API area at $254 \mathrm{~nm}$ on injection volume
The largest coefficient of linear regression was observed at $254 \mathrm{~nm}$, that is why we chose this wavelength (Fig. 6).

\section{Quantitative determination of API potassium \\ 2-((4-amino-5-(morpholinomethyl)-4H-1,2,4-triazol- 3 -yl)thio)acetate in the substance}

The solution of standard sample was chromatographed 5 times. The results are presented in Tables 2, 3. The obtained RSD value was in the requirements of the SPU to the $\mathrm{RSD} \%_{\text {max }}$ at all $\mathrm{n}$ values, starting with $\mathrm{n}=2$. Therefore, we chromatographed 2 times in turn of the reference solution and the research solution $[16,17]$. Chromatogram of research solution of potassium 2-((4-amino-5-(morpholinomethyl)4H-1,2,4-triazol-3-yl)thio)acetate (API) is shown in Fig. 7.

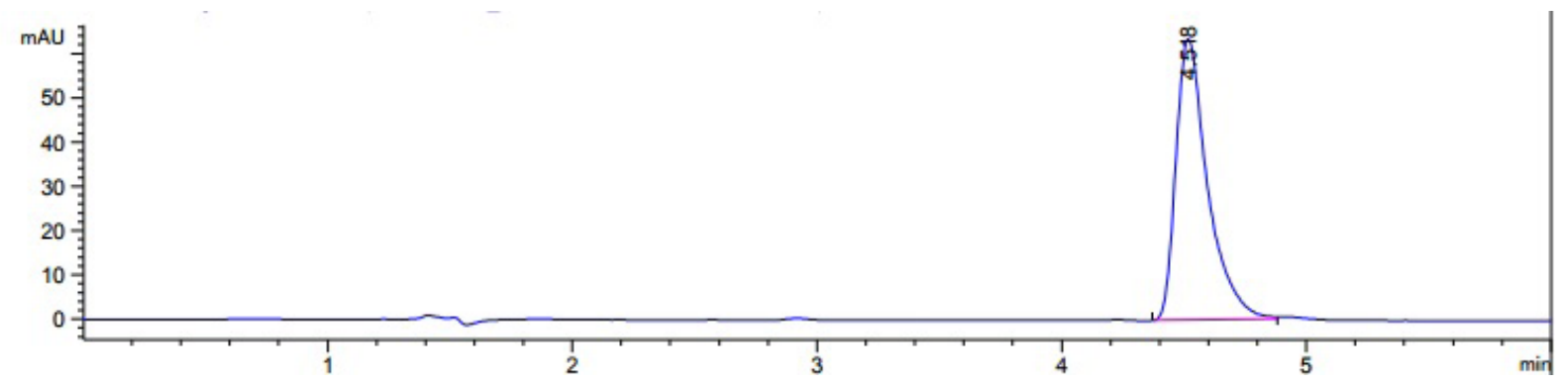

Figure 7. Chromatogram of research solution of potassium 2-((4-amino-5-(morpholinomethyl)-4H-1,2,4-triazol-3-yl)thio)acetate (API) 
Table 2. Results the chromatographic system suitability research of the reference solution for RSD

\begin{tabular}{|c|c|c|c|c|}
\hline № of chromatogram & Areast & mean Areast & RSD $\%$ & RSD \%max \\
\hline 1 & 540.47 & - & - & - \\
\hline 2 & 536.68 & 538.58 & 0.4974 & 0.32 \\
\hline 3 & 537.68 & 538.28 & 0.3648 & 0.84 \\
\hline 4 & 537.43 & 538.07 & 0.3083 & 1.20 \\
\hline 5 & 539.50 & 538.35 & 0.2926 & 1.48 \\
\hline
\end{tabular}

Area $_{\text {st }}$ - the peak area obtained by chromatography of the reference solution mean Area $_{\text {st }}$ - average peak area

RSD \% - relative standard deviation, \%

$\mathrm{RSD} \%_{\max }$ - maximal relative standard deviation, $\%$

Table 3. Results of quantitative determination of API potassium 2-((4-amino-5-(morpholinomethyl)-4H-1,2,4-triazol-3-yl)thio) acetate in the substance

\begin{tabular}{|c|c|c|c|c|c|}
\hline № & $\begin{array}{l}\text { Weight } \\
\text { (g) }\end{array}$ & \multicolumn{2}{|c|}{ The peak area } & $\begin{array}{l}\text { Found API } \\
\text { in } \%\end{array}$ & $\begin{array}{c}\text { Metrological } \\
\text { characteristic } \\
n-1=5\end{array}$ \\
\hline \multirow{2}{*}{1} & \multirow{2}{*}{0.1050} & 572.52 & \multirow{2}{*}{571.88} & \multirow{2}{*}{99.21} & \multirow{12}{*}{$\begin{aligned} \bar{X} & =100.9 \\
\mathrm{~S} & =1.369 \\
\mathrm{~S} & =1.357 \\
\Delta \overline{\mathrm{X}} & =1.437 \\
\varepsilon & =1.424 \%\end{aligned}$} \\
\hline & & 571.25 & & & \\
\hline \multirow{2}{*}{2} & \multirow{2}{*}{0.1153} & 626.85 & \multirow{2}{*}{627.15} & \multirow{2}{*}{99.07} & \\
\hline & & 627.45 & & & \\
\hline \multirow{2}{*}{3} & \multirow{2}{*}{0.1088} & 606.64 & \multirow{2}{*}{608.97} & \multirow{2}{*}{101.9} & \\
\hline & & 611.30 & & & \\
\hline \multirow{2}{*}{4} & \multirow{2}{*}{0.1035} & 579.40 & \multirow{2}{*}{579.14} & \multirow{2}{*}{101.9} & \\
\hline & & 578.89 & & & \\
\hline \multirow{2}{*}{5} & \multirow{2}{*}{0.1099} & 610.86 & \multirow{2}{*}{612.60} & \multirow{2}{*}{101.5} & \\
\hline & & 614.34 & & & \\
\hline \multirow{2}{*}{6} & \multirow{2}{*}{0.1190} & 670.07 & \multirow{2}{*}{665.22} & \multirow{2}{*}{101.8} & \\
\hline & & 660.36 & & & \\
\hline \multirow{2}{*}{$\begin{array}{c}\text { Reference } \\
\text { solution }\end{array}$} & \multirow{2}{*}{0.0980} & 540.47 & \multirow{2}{*}{538.58} & & \\
\hline & & 536.68 & & & \\
\hline
\end{tabular}

\section{CONCLUSIONS}

Due to the development of a method for determination of the API in the potassium 2-((4-amino-5-(morpholinomethyl)4H-1,2,4-triazol-3-yl)thio)acetate, the substance was researched using the HPLC-DAD method. From the study of the API sample, the admixture of the original component of 4-amino-5-(morpholinomethyl)-4H-1,2,4-triazole-3-thiol was identified. The results of determining the contents of the API in real samples indicate that the method can be proposed to control the quality of the substance potassium 2-((4-amino-5-(morpholinomethyl)-4H-1,2,4-triazol-3-yl) thio)acetate.

\section{ORCID iDs}

Roman Shcherbyna Dhttps://orcid.org/0000-0002-9742-0284 Volodymyr Parchenko (Dhttps://orcid.org/0000-0002-2283-1695 Boris Varynskyi Dhttps://orcid.org/0000-0002-1551-8879 Andriy Kaplaushenko (Dhttps://orcid.org/0000-0003-3704-5539

\section{REFERENCES}

1. Blumenthal GM, Goldberg KB, Pazdur R. Drug development, trial design, and endpoints in oncology: adapting to rapidly changing science. Clinical Pharmacology \& Therapeutics. 2017;101:572-4.
2. Dimitrios Z, Pridgen CS, Vincenzo L. Process development and robust control of physical attributes of an amorphous drug substance. Journal of Pharmaceutical Sciences. 2018;107:217-30.

3. Sandeep S, Vohora D. Drug discovery and development: An overview. Pharmaceutical Medicine and Translational Clinical Research. 2018;19:19-32.

4. Bulut N, Kocyigit UM, Gecibesler IH, Dastan T, Karci H, Taslimi P et al. Synthesis of some novel pyridine compounds containing bis 1,2,4-triazole/thiosemicarbazide moiety and investigation of their antioxidant properties, carbonic anhydrase, and acetylcholinesterase enzymes inhibition profiles. Journal of Biochemical and Molecular Toxicology. 2018;32: 1-10.

5. Kumar R, Shahar Yar M, Chaturvedi S, Srivastava A. Triazole as pharmaceuticals potentials. Int. J. pharm. Tech. Res. 2013;5:1844-69.

6. El-Gazzar YI, Georgey HH, El-Messery SM, Ewida HA, Hassan GS, Raafat MM, et al. Synthesis, biological evaluation and molecular modeling study of new (1,2,4-triazole or 1,3,4-thiadiazole)methylthio-derivatives of quinazolin-4(3H)-one as DHFR inhibitors. Bioorganic Chemistry. 2017; 72: 282-92.

7. Shcherbyna RO, Danilchenko DM, Parchenko VV, Panasenko OI, Knysh EH, Hromyh NA, et al. Studying of 2-((5-R-4-R1-4H-1,2,4triazole-3-yl)thio)acetic acid salts influence on growth and progress of blackberries (KIOWA Variety) propagules. Res. J. Pharm., Biol. Chem. Sci. 2017;8: 975-9.

8. Singala P, Talpara P. Synthesis of new 1,2,4-triazole derivatives and their antimicrobial screening. International Journal of Scientific Research in Science, Engineering and Technology. 2017;3:1009-12.

9. Shcherbyna RO, Samura TO, Kyrychko BP, Zvenihorodska TV, Hyrenko IV. The research of ammonium 2-((4-amino-5(morpholinomethyl)-4H-1,2,4-triazole-3-yl)thio)acetate (PKR177) influence on biochemical indices in rats blood under hepatitis initiated by tetrachloride methane. Zaporozhye Medical Journal. 2017; 19:819-22.

10. Shcherbyna RO, Panasenko OI, Knysh YG, Fotina HA, Vashchyk YV, Fotina TI. The study of antimicrobial activity of 2-((4-R-3(morpholinomethylene)-4H-1,2,4-triazole-5-yl)thio)acetic acid salts. Zaporozhye Medical Journal. 2016;4:97-0.

11. Shcherbyna RO, Panasenko OI, Knysh YeH. The studying of antioxidant activity of salts 2-((4-R-3-(morpholinomethylen)- $4 \mathrm{H}$ 1,2,4-triazole-5-yl)thio)acetic acids. Ukraïns'kij Biofarmacevtičnij Žurnal. 2016;42: 37-0.

12. Anderson J, Gerlin M, Sversut R, Oliveira L, Singh A, Amaral M et al. Development and validation of an isocratic HPLC method for simultaneous determination of quaternary mixtures of antihypertensive drugs in pharmaceutical formulations. Acta Chromatographica. 2017;29:95-10.

13. Baker M, El-Kafrawy D, Mahrous M, Belal T. Validated stabilityindicating HPLC-DAD method for determination of the recently approved hepatitis C antiviral agent daclatasvir. Annales Pharmaceutiques Françaises. 2017;75:176-4.

14. Pavlović D, Babić S, Horvat A, Kaštelan-Macan M. Sample preparation in analysis of pharmaceuticals. TrAC Trends in Analytical Chemistry. 2007;26:1062-5.

15. Shcherbyna RO. Synthesis, physical and chemical properties of 2-((4-R-3-(morfolinomethylen)-4H-1,2,4-triazole-5-yl)thio)acetic acid salts. Current issues in pharmacy and medicine: science and practice. 2017;10: 4-8.

16. State Pharmacopoeia of Ukraine. $1^{\text {st }}$ ed. Kharkiv: DP "Naukovoekspertnii farmakopeinii tsentr";2001:538.

17. State Pharmacopoeia of Ukraine. Supplement 1. $2^{\text {nd }}$ ed. Kharkiv: RIREKH; 2004: 494.

18. European pharmacopoeia. Strasbourg: Council of Europe; 2016

19. Direct injection electrospray mass spectrometry using Agilent 1100 Series LC-MS [Internet]. Haverford; 2018 [cited 29 March 2018]. Available from: https://www.haverford.edu/sites/default/files/ Department/Chemistry/Agilent\%20Direct\%20Inject.pdf 